\title{
Regulated fractionally integrated processes
}

\section{Mirza Trokićc ${ }^{*, \dagger}$}

\begin{abstract}
Regulated (bounded) integrated time series are of significant practical importance and a recent development in the time series literature. Although regulated integrated series are characterized by asymptotic distributions that differ substantially from their unregulated counterparts, most inferential exercises continue to be performed with complete disregard for this potential feature of time series data. To date, only Cavaliere (2005) and Cavaliere and Xu (2011) have attempted to develop a theory for regulated integrated time series, particularly in the context of unit root testing. Unfortunately, no such theory has been developed for regulated fractionally integrated series, which are particularly important in financial time series and also in some unit root testing literature. This article achieves just this: it establishes a framework for regulated fractionally integrated processes and develops their functional central limit distributions. In addition, this article presents some simulation evidence and discusses several algorithms for obtaining the limiting distributions for these processes.
\end{abstract}

Keywords: Regulated time series; fractionally integrated time series; fractional Brownian motion

\section{INTRODUCTION}

Recently, the introduction of 'regulated' (bounded) integrated time series to econometrics has been an exciting development. To date, very little has been written about it, and as such, it is proving to be a fruitful terrain for theoreticians and practitioners alike. To shed light on what is meant by such processes, Granger (2010) notes that 'a limited process is one that has bounds either below (at zero, say) or above (full capacity) or both'. The issue however is that despite the fact that traditional integration methods fail in the presence of regulated integrated processes, the latter are almost always modelled as pure I(1) processes. Indeed, regulated series such as nominal interest rates, target zone exchange rates, unemployment rates and many others are prevalent in econometrics, and as such, we ought to model them correctly.

At the moment, Cavaliere (2005), Granger (2010) and Cavaliere and Xu (2011) are the only serious efforts to develop a theory for regulated integrated processes. Particularly important is the seminal article of Cavaliere (2005) in which he develops asymptotic distributions for well-known unit root test statistics when the driving series is a regulated I(1) process. More importantly, he demonstrates that the latter have distributions that are characterized by functionals of the regulated Brownian motion (see Harrison, 1985) and as such depend on nuisance parameters that are determined by the bounds of the process. These results are further developed in Cavaliere and $\mathrm{Xu}$ (2011) by allowing for constant deterministic components in addition to specifying errors as general linear processes of martingale difference innovations.

Despite these promising developments, Cavaliere and Xu (2011) are still only concerned with bounded I(1) processes and have not considered more general scenarios involving integrated fractional processes such as $\mathrm{I}(d)$. The latter are a very important subject in time series analysis particularly when $d$ is taken to be some real number in the range $(-1 / 2,1)$. Such processes are known as fractionally integrated processes and are themselves a very popular and modern research topic. In this regard, this article aims to bridge the gap in the literature by meshing the development of bounded integrated time series with fractionally integrated processes.

The article is organized as follows. The next section will develop a model of bounded fractionally integrated processes as well as outline the assumptions necessary to prove the main results of the article. Section 3 will derive the main results. Finally, Section 4 will discuss empirical implications with simulation evidence.

\section{REGULATED FRACTIONALLY INTEGRATED PROCESSES}

We will establish a relatively general class of regulated fractionally integrated processes. Such processes are I $(d)$ and are regulated so as to have bounds above, below or both. In the case of $d=1$, Cavaliere and $\mathrm{Xu}(2011)$ refer to these series as bounded I(1), or $\mathrm{BI}(1)$ 
series. Such notation however is too reminiscent of the notation used to denote Brownian motion processes. For this reason, to avoid the possible association, denote bounded $\mathrm{I}(d)$ processes as regulated $\mathrm{I}(d)$ processes, or $\mathrm{RI}(d)$ in short. For cases when $d$ is not an integer, $\mathrm{I}(d)$ processes are referred to as fractionally integrated of order $d$. Accordingly, when such processes are regulated, they will be referred to as regulated fractionally integrated processes of order $d$, or $\operatorname{RFI}(d)$ in short.

A general fractionally integrated process $\left\{z_{t}\right\}$ of order $d$ is defined as

$$
(1-B)^{d} z_{t}=u_{t}, \quad \sum_{j=0}^{\infty} \psi_{j} \epsilon_{t-j}, \quad t=1,2, \ldots
$$

where $d>-1 / 2, \epsilon_{t}$ are zero-mean, finite variance, i.i.d. random variables, $B$ is the backshift operator and $(1-B)^{d}$ is defined by the Maclaurin series:

$$
(1-B)^{d}=\sum_{j=0}^{\infty} \frac{\Gamma(-d+j)}{\Gamma(-d) \Gamma(j+1)} B^{j} .
$$

If we now assume that only terms with a positive time index are of interest, as is the case in practice, then it can be shown (see Appendix 2 of Wang et al. (2002)) that $z_{t}$ reduces to

$$
z_{t}=(1-B)_{+}^{-d} u_{t} \equiv \Delta_{+}^{-d} u_{t}=\sum_{k=0}^{t-1} c_{k}^{(d)} u_{t-k}
$$

where

$$
c_{k}^{(d)}=\frac{\Gamma(d+k)}{\Gamma(d) \Gamma(k+1)}, k \geq 0, c_{0}^{(0)}=1, c_{k}^{(0)}=0, k \geq 1
$$

On the other hand, a regulated series $x_{t}$ with fixed bounds at $\underline{b}$ and $\bar{b}$ with $\underline{b}<\bar{b}$ satisfies the condition $x_{t} \in[\underline{b}, \bar{b}]$ almost surely for all $t$. To develop then the idea of a bounded fractionally integrated process, the framework in Cavaliere and Xu (2011) with a constant deterministic trend $\gamma$ and a general linear process of martingale difference innovations is meshed with the concepts presented earlier. In particular, let the underlying process be a fractionally integrated process of order $d>-1 / 2$ rather than I(1) and assume that prehistoric treatment of the $\mathrm{I}(d)$ process is such that only terms with a positive time index enter into the series. Accordingly, consider the following setup:

$$
\begin{aligned}
& y_{t}=\phi y_{t-1}+z_{t}, \phi=1 \\
& z_{t}=\Delta_{+}^{-d} u_{t} \\
& u_{t}=v_{t}+\underline{\xi}_{d, t}-\bar{\xi}_{d, t} \\
& x_{t}=\gamma+y_{t} .
\end{aligned}
$$

In the equations, $\underline{\xi}_{d, t} \equiv \Delta_{+}^{d} \underline{\xi}_{t}$ and $\bar{\xi}_{d, t} \equiv \Delta_{+}^{d} \bar{\xi}_{t}$. Following the terminology of Harrison (1985), $\underline{\xi}_{t}$ and $\bar{\xi}_{t}$ are regulators that ensure that $x_{t} \in[\underline{b}, \bar{b}]$. Moreover, they are both non-negative and satisfy the following relations:

$$
\begin{aligned}
& \underline{\xi}_{t}>0 \text { iff } y_{t-1}+z_{t}<\underline{b}-\gamma \\
& \bar{\xi}_{t}>0 \text { iff } y_{t-1}+z_{t}>\bar{b}-\gamma .
\end{aligned}
$$

In addition, to allow for general covariance structures in the error terms, assume that $v_{t}$ is a general linear process of the form

$$
v_{t}=\Psi(L) \epsilon_{t} \text { where } \Psi(z) \equiv \sum_{j=0}^{\infty} \psi_{j} z^{j}
$$

and $\epsilon_{t}$ is a martingale difference sequence. Also, make note of the following assumptions that will be used throughout the remainder of the article: 


\section{Assumptions}

(a) $\left\{\epsilon_{t}, \mathcal{F}_{t}\right\}$ is a martingale difference sequence with respect to some filtration $\mathcal{F}_{t}$.

(b) $E\left\{\epsilon_{t}^{2}\right\}=\sigma^{2}<\infty$ and $E\left\{\left|\epsilon_{0}\right|^{2 /(2 d+1)}\right\}<\infty$ for $d>-1 / 2$.

(c) $\sum_{j=0}^{\infty}\left|\psi_{j}\right|<\infty, \sum_{j=0}^{\infty} j\left|\psi_{j}\right|<\infty$, and $b_{\psi}=\sum_{j=0}^{\infty} \psi_{j} \neq 0$

(d) $\sup _{t} E\left\{\left|\underline{\xi}_{t}\right|^{2 /(2 d+1)}\right\}<\infty$ and $\sup _{t} E\left\{\left|\bar{\xi}_{t}\right|^{2 /(2 d+1)}\right\}<\infty$, for $d>-1 / 2$

(e) $\left(\left(\frac{b_{\psi} \sigma}{\Gamma(d+1)}\right)^{2} T^{2(d+1 / 2)}\right)^{-1 / 2}(\underline{b}-\gamma)=\underline{c}+o(1),\left(\left(\frac{b_{\psi} \sigma}{\Gamma(d+1)}\right)^{2} T^{2(d+1 / 2)}\right)^{-1 / 2}(\bar{b}-\gamma)=\bar{c}+o(1)$ where $c \leq 0 \leq \bar{c}$ and $\underline{c} \neq \bar{c}$, and $d>-1 / 2$.

(f) $\frac{1}{k}+\frac{k^{2}}{T} \rightarrow 0$ as $T \rightarrow \infty$.

A quick reflection on the assumptions is in order. Assumption (a) and the first part of assumption (b) is in fact assumption $\mathcal{A}_{1}(\mathrm{a})$ in Cavaliere and $\mathrm{Xu}$ (2011). Here, $\lambda^{2} \equiv \Psi^{2}(1) \sigma^{2}=b_{\psi}^{2} \sigma^{2}$ is in fact the long-run variance of $v_{t}$, and under assumption (b), $\Psi^{-1}(z)$ is well defined. Note however that the latter part of assumption (b) is much weaker than assumption $\mathcal{A}_{1}(\mathrm{c})$ in Cavaliere and Xu (2011). There, following the article by Chang and Park (2002), the authors require that $E\left\{\left|\epsilon_{t}\right|^{r}\right\}<\infty$ for $r>4$. However, as discussed in Wang et al. (2002), for the purpose set out here, this assumption can be significantly weakened to the one stated earlier. Assumption (c) is the same as assumption $\mathcal{A}_{2}$ in Cavaliere and Xu (2011) and is also required by Wang et al. (2002). Assumptions (d) and (e) are similar but different from assumptions $\mathcal{B}_{1}$ and $\mathcal{B}_{2}$ in Cavaliere and $\mathrm{Xu}$ (2011). This is to accommodate the fractional integration setting of this article and is a natural extension.

Note further that asymptotically, the presence of the non-zero deterministic term $\gamma$ in eqn (4) is handled gracefully by assumption (e). Essentially, the assumption establishes the relationship between series bounds $\underline{b}$ and $\bar{b}$ and distributional bounds $\underline{c}$ and $\bar{c}$ respectively, relative to the true mean $\gamma$ of $x_{t}$. Here, of course, $\gamma$ is assumed known. When $\gamma$ needs to be estimated however, techniques such as OLS or GLS demeaning as in Elliott et al. (1996) are popular procedures for handling unknown location parameters. To reflect such frameworks in the asymptotic results obtained later, functionals of regulated fractional Brownian motions would have to be replaced by their demeaned analogues.

The following definition formalizes what is meant by a regulated fractionally integrated process. It parallels Definition 1 of Cavaliere (2005) and is a starting point for the subsequent analysis.

Definition 1: A process $\left\{x_{t}\right\}$ satisfying equations (1)-(6) and assumptions (a)-(e) will be called a generalized regulated fractionally integrated process of degree $d$, or a generalized $\mathrm{RFI}(d)$ process in short.

\section{ASYMPTOTIC ANALYSIS OF THE RFI(D) PROCESS}

The asymptotic analysis of the $\mathrm{RFI}(d)$ process will rely heavily on the theory developed by Cavaliere (2005) for regulated I(1) processes and the theory developed by Wang et al. (2002) for general non-stationary fractionally integrated processes. These works will be meshed to develop the limiting distribution of the regulated fractionally integrated process.

Recall that a type II fractional Brownian motion $B_{d}(t)$ for $d>-1 / 2$ is defined as follows:

$$
B_{d}(t)=\int_{0}^{t}(t-s)^{d-1} d W(s), 0 \leq t \leq 1, B_{d}(0)=0 .
$$

For a good survey on the difference between type I and type II fractional Brownian motions, see Davidson and Hashimzade (2009). For weak convergence of multivariate fractionally integrated processes, see Marinucci and Robinson (2000). Now, consider the following result from Harrison (1985), which lies at the heart of the main result of this article.

Lemma 1: Fix the limits $[\underline{b}, \bar{b}]=[0, b]$ with $b>0$ and let $\mathcal{C}$ be the space of all continuous functions. Define $\mathcal{C}_{0}$ as the set of all functions $x \in \mathcal{C}$ such that $x_{0} \in[0, b]$. Then, for each $x \in \mathcal{C}_{0}$, there is a unique pair of continuous functions $(I, q)$, which satisfy $I_{t}=\sup _{0 \leq s \leq t}\left(x_{s}-q_{s}\right)^{-}$and $q_{t}=\sup _{0 \leq s \leq t}\left(b-x_{s}-I_{s}\right)^{-}$, and this same pair satisfies the following three properties:

(1) $I_{t}$ and $q_{t}$ are continuous and increasing and satisfy $I_{0}=q_{0}=0$.

(2) $h_{t} \equiv\left(x_{t}+l_{t}-q_{t}\right) \in[0, b]$ for all $t \geq 0$.

(3) $I_{t}$ and $q_{t}$ increase only when $h_{t}=0$ and $h_{t}=b$ respectively. 
Proof : Lemma 1 is Proposition 2.4.6 in Harrison (1985).

Since $B_{d}(t) \in \mathcal{C}$, Lemma 1 tells us that we can find a pair of functions $/$ and $u$ so that $B_{d}(t)+I(t)-q(t)$ is regulated process. Formally,

Definition 2: Let $w_{d}(t)=B_{d}(t)$ be a stochastic process on $\mathcal{C}$. Fix the bounds $\underline{b}$ and $\bar{b}$. If $w_{d}(0) \in[\underline{b}, \bar{b}]$, then there exist continuous functions $I(t)$ and $q(t)$ satisfying the conditions of Lemma 1 such that the process $B_{d}^{\underline{b}, \bar{b}}(t) \equiv\left(w_{d}(t)+I(t)-q(t)\right) \in$ $[\underline{b}, \bar{b}]$. The functions $I(t)$ and $q(t)$ are called regulators, and the function $B_{d}^{\underline{b}, \bar{b}}(t)$ will be called a 'regulated type $I$ fractional Brownian motion' with bounds at $[\underline{b}, \bar{b}]$.

Definition 2 extends the Definition 2 of Cavaliere (2005) to the case when the underlying process is modelled as a type II fractional Brownian motion. Next, let $\Rightarrow$ denote weak convergence and consider the following lemmas.

LemmA 2: Let $w_{t}$ satisfy $\Delta_{+}^{d} w_{t}=v_{t}$, with $v_{t}=\sum_{k=0}^{\infty} \psi_{k} \epsilon_{t-k}$ for $t=1,2, \ldots$. Assume conditions $(a)-(c)$ hold and define $\kappa(d)=\frac{b_{\psi} \sigma}{\Gamma(d+1)^{*}}$. Then, for $d>-1 / 2$,

$$
\left(\kappa^{2}(d) T^{2(d+1 / 2)}\right)^{-1 / 2} \sum_{j=1}^{\left[T_{t}\right]} w_{t} \Rightarrow \int_{0}^{t}(t-s)^{d} d W(s), 0 \leq t \leq 1 .
$$

Proof : Lemma 2 is Corollary 2 in Wang et al. (2002).

Consider next the continuous-time approximation of a $\operatorname{RFI}(d)$ process $\left\{x_{t}\right\}$ on the cadlag space $\mathcal{D}[0,1]$

$$
x_{T}(t)=\left(\kappa^{2}(d) T^{2(d+1 / 2)}\right)^{-1 / 2}\left(x_{[T t]}-x_{0}\right), t \in[0,1]
$$

Note that approximations of the sort above can always be constructed by transforming the original series through the broken line process. The main result of this article is now summarized in the following theorem.

THEOREM 1: Let $x_{t}$ be $R F I(d)$ and fix the bounds $[\underline{b}, \bar{b}]$. If $\mathcal{D}[0,1]$ is endowed with the uniform topology and $x_{0} \in[\underline{c}, \bar{c}]$, then $x_{T}(t) \Rightarrow B_{d+1}^{\underline{c}, \bar{c}}(t)$ for any $d>-1 / 2$.

Proof : See proof in the Appendix.

Recall from the discussion in Section 2 that Theorem 1 can accommodate a non-zero unknown $\gamma$ through OLS or GLS demeaning. Typically, $x_{t}$ would be replaced by residuals from the demeaning regression and $B_{d+1}^{c, \bar{c}}(t)$ would be replaced by the demeaned regulated type II fractional Brownian motion that, under OLS demeaning, takes the form $\widetilde{B}_{d+1}^{\underline{c}, \bar{c}}=B_{d+1}^{\frac{c}{, c} \bar{c}}(t)-\int_{0}^{1} B_{d+1}^{\underline{c}, \bar{c}}(s) d s$.

\section{EMPIRICAL CONSIDERATIONS}

A critical feature of the construction developed in this article, which is in fact shared by the articles by Cavaliere (2005) and Cavaliere and $\mathrm{Xu}(2011)$, is that the bounds $[\underline{b}, \bar{b}]$ are known a priori. Moreover, this article introduces an additional nuisance parameter through the fractional co-integration parameter $d$. It too is assumed known. This has significant empirical implications for $[\underline{c}, \bar{c}]$ as it means that the latter can be estimated consistently.

Start by considering again assumption (e).

$$
\begin{aligned}
& \left(\frac{\lambda^{2}}{\Gamma^{2}(d+1)} T^{2(d+1 / 2)}\right)^{-1 / 2}(\underline{b}-\gamma)=\underline{c}+o(1) \\
& \left(\frac{\lambda^{2}}{\Gamma^{2}(d+1)} T^{2(d+1 / 2)}\right)^{-1 / 2}(\bar{b}-\gamma)=\bar{c}+o(1),
\end{aligned}
$$

where $\underline{c}<0<\bar{c}, \underline{c} \neq \bar{c}$ and $d>-1 / 2$, and as mentioned earlier, $\lambda^{2}=b_{\psi}^{2} \sigma^{2}$ is the long-run variance of $v_{t}$. Since $[\underline{b}, \bar{b}]$ and $d$ are known, it follows that if $\lambda^{2}$ can be estimated consistently, one can obtain a means for simulating a time series with a distribution that asymptotically approaches the regulated fractionally integrated Brownian motion defined earlier. In this regard, Cavaliere and $\mathrm{Xu}$ (2011) propose the use of the widely popular autoregressive spectral density estimator: 


$$
s_{A R}^{2} \equiv \frac{\hat{\sigma}^{2}}{\left(\hat{b}_{\psi}^{-1}\right)^{2}}=\frac{\hat{\sigma}^{2}}{\hat{a}_{\psi}^{2}}
$$

where $a_{\psi} \equiv \psi^{-1}(1)$. Cavaliere and Xu (2011) demonstrate the consistency of this estimator even in the presence of regulators, and the context of this article does not alter their result.

Lemma 3: Define the following estimators:

$$
\begin{aligned}
& \hat{\underline{c}}=\left(\frac{s_{A R}^{2}}{\Gamma^{2}(d+1)} T^{2(d+1 / 2)}\right)^{-1 / 2}(\underline{b}-\gamma) \\
& \hat{\bar{c}}=\left(\frac{s_{A R}^{2}}{\Gamma^{2}(d+1)} T^{2(d+1 / 2)}\right)^{-1 / 2}(\bar{b}-\gamma)
\end{aligned}
$$

Provided that $[\underline{b}, \bar{b}]$ and $d$ are known, $\underline{\hat{c}} \rightarrow^{P} \underline{c}$ and $\hat{\bar{c}} \rightarrow^{P} \bar{c}$.

Proof : This is Lemma 1 of Cavaliere and Xu (2011) with appropriate notation changes.

The above naturally leads to the following result, which also describes a means for simulating regulated fractionally integrated processes.

THEOREM 2: Let $\left\{x_{t}^{*}\right\}$ be defined through the following recursion:

$$
x_{t}^{*}= \begin{cases}\hat{\bar{c}} & \text { if } x_{t-1}^{*}+\left(\kappa(d) T^{(d+1 / 2)}\right)^{-1} z_{t}^{*}>\hat{\bar{c}} \\ \hat{c} & \text { if } x_{t-1}^{*}+\left(\kappa(d) T^{(d+1 / 2)}\right)^{-1} z_{t}^{*}<\hat{c} \\ x_{t-1}^{*}+\left(\kappa(d) T^{(d+1 / 2)}\right)^{-1} z_{t}^{*} & \text { otherwise }\end{cases}
$$

where $\left\{z_{t}^{*}\right\}$ is a general linear processes (possibly satisfying eqn (7)), which is drawn independently of $\left\{x_{t}^{*}\right\}$. Let Assumption 1 hold and assume that $x_{0}^{*} \in[\underline{c}, \bar{c}]$. Then, as $T \rightarrow \infty, x_{\lfloor T t]}^{*} \Rightarrow B_{d+1}^{\underline{c}, \bar{c}}(t)$ for $t \in[0,1]$ and $d>-1 / 2$.

Proof of Theorem 2 : The result follows immediately from Theorem 1 and virtually the same strategy used in the proof of Theorem 2 in Cavaliere and Xu (2011).

\subsection{Simulation evidence}

Abstracting for the moment from estimation procedures, Theorem 1 says that if starting with an I(1) process $y(t)$ regulated at $[\underline{b}, \bar{b}]$, the partial sum process $x(t)=\gamma+y(t)$ is distributed as a regulated fractionally integrated Brownian motion $B_{d+1}^{c, \bar{c}}(t)$, where

$$
\begin{aligned}
& \left(\left(\frac{b_{\psi} \sigma}{\Gamma(d+1)}\right)^{2} T^{2(d+1 / 2)}\right)^{-1 / 2}(\underline{b}-\gamma)=\underline{c} \\
& \left(\left(\frac{b_{\psi} \sigma}{\Gamma(d+1)}\right)^{2} T^{2(d+1 / 2)}\right)^{-1 / 2}(\bar{b}-\gamma)=\bar{c} .
\end{aligned}
$$

Theorem 2 on the other hand provides an algorithm for simulating said regulated fractionally integrated processes. It is described as follows:

\section{Algorithm 1:}

(1) Fix the sample size $T$ and fractional integration parameter $d$.

(2) Fix the lower bound $\underline{b}$ and the upper bound $\bar{b}$ of the original series $y_{t}$.

(3) Compute the bounds $[\underline{c}, \bar{c}]$ which will regulate the simulated series using eqns (11) and (12).

(4) Generate innovations $\left\{\zeta_{1}, \ldots, \zeta_{T}\right\}$ (independent of all other series) from some ARMA processes satisfying assumptions (b) and (c) above.

(5) Use equation (1) to compute the fractional partial sums $\left\{\eta_{1}, \ldots \eta_{T}\right\}$ where $\eta_{t}=\Delta_{+}^{-d} \zeta_{t}$.

(6) Use equation (10) to compute the simulated fractionally integrated series $x_{t}^{*}$. 
According to Theorem 1 , as $T \rightarrow \infty$, the cumulated sums $x_{\lfloor T t\rfloor}^{*}$ approach the regulated fractionally integrated process $B_{d+1}^{\frac{c}{c} \bar{c}}(t)$. To demonstrate the algorithm, several simulations were performed and presented in the following graphs. Each simulation was performed on a sample size $T=2^{13}$ using standard normal i.i.d. innovations. Although a higher sample size could have been chosen as computing time is not very expensive, the graphs would have become too cluttered to be useful. As expected, the simulated series approach their expected distributions. Note that in Figures 1 and 2, the graph pertaining to $x_{T}(t)$ is plotted using an evenly spaced sample of 300 points from the original data. This was carried out for aesthetic purposes as all three graphs overlap, as they should.

An issue that was not discussed until now is the functional nature of the regulator functions $\underline{\xi}_{t}$ and $\bar{\xi}_{t}$. Thus far, the latter were assumed to be the simple censoring functions acting on their inputs to restrict values to the $[\underline{b}, \bar{b}]$ interval. However, as Cavaliere (2005) points out, there are other possibilities. A particularly important alternative is the reflection function. The concept is more commonly known as the reflection principle in the Brownian motion literature, and it states that, given a standard Brownian motion $B(t)$ and some stopping time $T$, then the function

$$
B^{*}(t)=B(t) \mathbb{I}_{\{t \leq T\}}+(2 B(T)-B(t)) \mathbb{I}_{\{t \leq T\}}
$$

is again a standard Brownian motion. However, because the limits $[\underline{c}, \bar{c}]$ correspond to some stopping times, this suggests that one can define the regulator functions to reflect a process at the points $[\underline{c}, \bar{c}]$ rather than censor it. This can be carried out by defining the lower and upper regulator functions as follows:

$$
\begin{aligned}
& \underline{\xi}_{i}=\left(2 \underline{c}-\left(x_{t-1}+z_{t}\right) \mathbb{I}_{\left\{x_{t-1}+z_{t}<\underline{c}\right\}}\right. \\
& \bar{\xi}_{i}=\left(2 \bar{c}-\left(x_{t-1}+z_{t}\right) \mathbb{I}_{\left\{x_{t-1}+z_{t}>\bar{c}\right\}}\right.
\end{aligned}
$$

From an algorithmic perspective, it turns out that the choice of the regulator function does not alter any of the results established so far. To see this, consider the following algorithm:

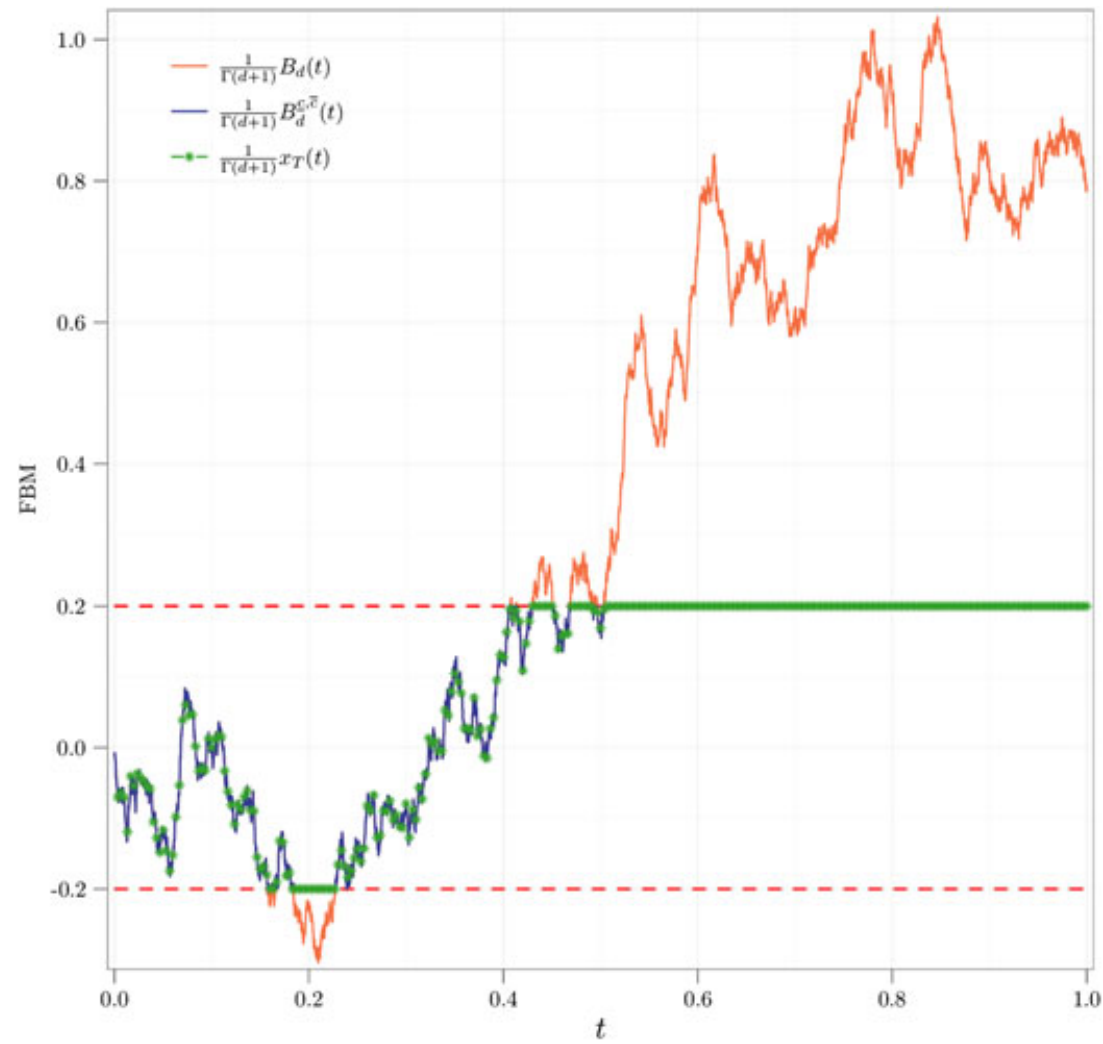

Figure 1. Regulated fractional Brownian motions (FBM): the graph is generated with a sample of size $2^{13}, d=0.1$, and asymptotic absorbing distributional bounds $\underline{c}=-0.2$ and $\bar{c}=0.2$ 


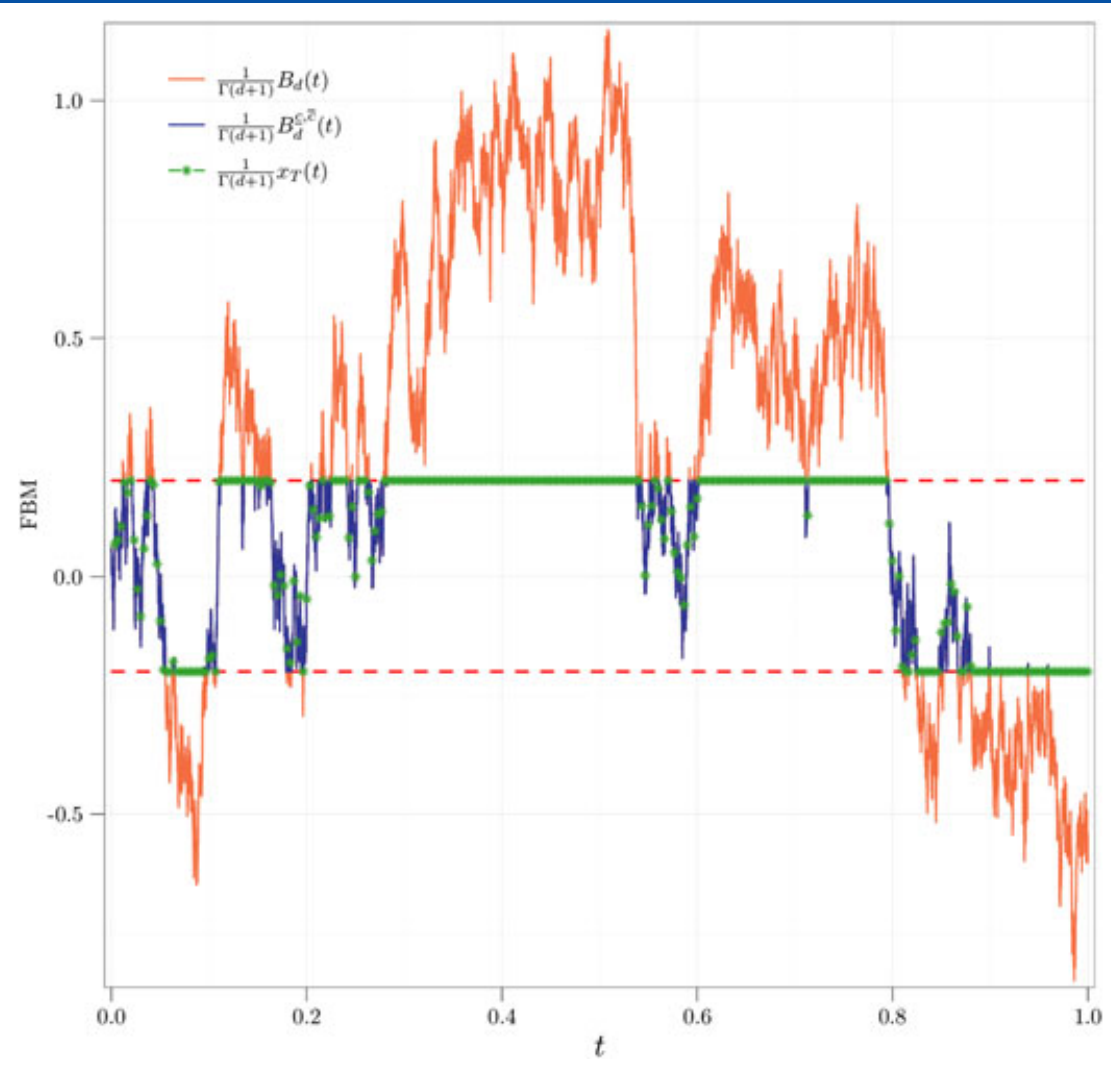

Figure 2. Regulated fractional Brownian motions (FBM): the graph is generated with a sample of size $2^{13}, d=-0.1$, and asymptotic absorbing distributional bounds $\underline{c}=-0.2$ and $\bar{c}=0.2$

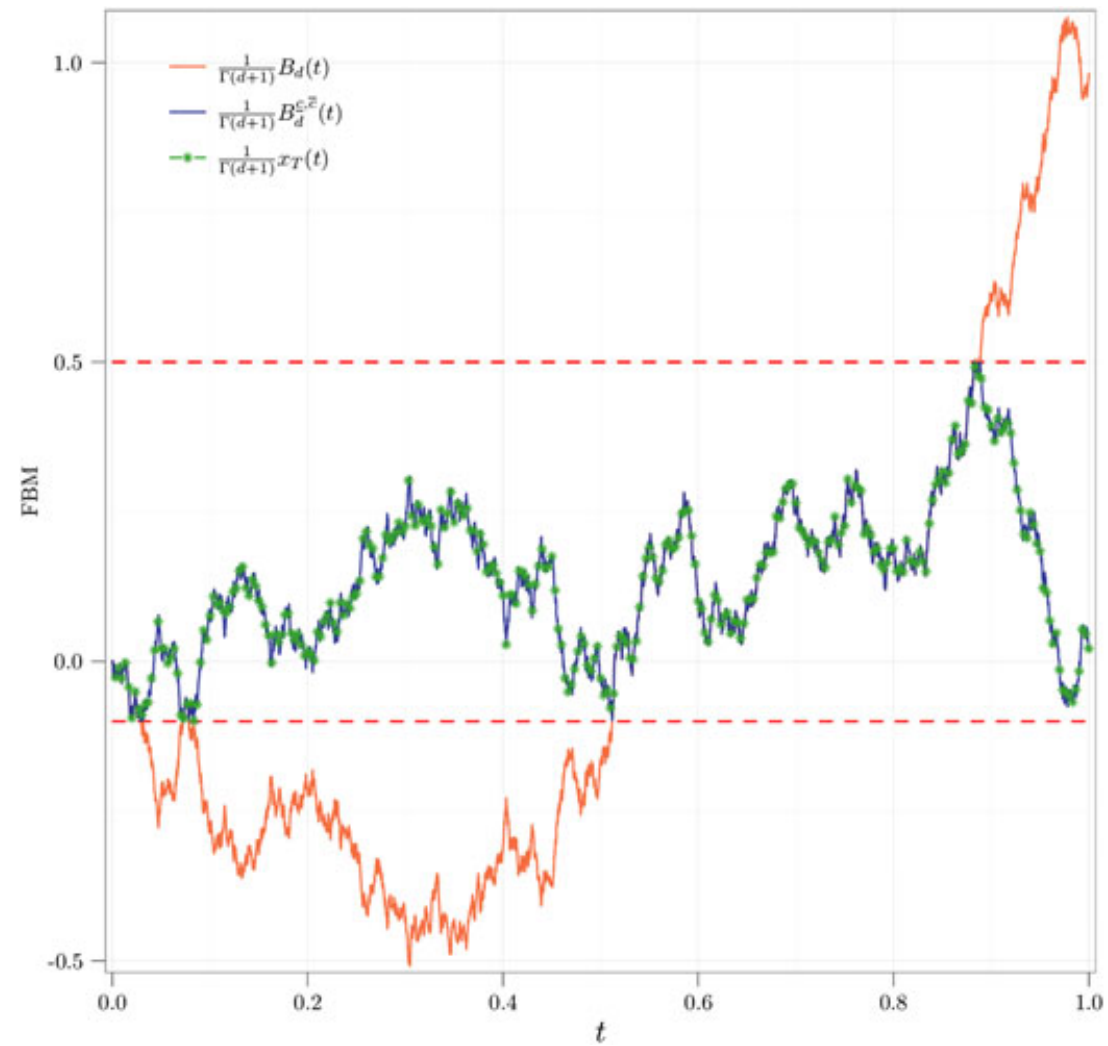

Figure 3. Regulated fractional Brownian motions (FBM): the graph is generated with a sample of size $2^{13}, d=0.1$, and asymptotic reflective distributional bounds $\underline{c}=-0.1$ and $\bar{c}=0.5$ 


\section{Algorithm 2:}

(1) Replicate steps 1 to 5 from Algorithm 1.

(2) Simulate a reflected fractionally integrated series using the following formula:

$$
x_{t}^{(1)}= \begin{cases}2 \hat{\bar{c}}-\left(x_{t-1}^{(1)}+\eta_{t}\right) & \text { if } x_{t-1}^{(1)}+\eta_{t}>\hat{\bar{c}} \\ 2 \hat{\hat{c}}-\left(x_{t-1}^{(1)}+\eta_{t}\right) & \text { if } x_{t-1}^{(1)}+\eta_{t}<\underline{\hat{c}} \\ x_{t-1}^{(1)}+\eta_{t} & \text { otherwise. }\end{cases}
$$

(3). Because the reflected process $x_{t}^{(1)}$ may exceed the desired bounds as a result of very lengthy shadow paths, it may be necessary to continue reflect the process again, however, this time, reflect the series according to the following formula:

$$
x_{t}^{(2)}= \begin{cases}2 \hat{\bar{c}}-\left(x_{t}^{(1)}\right) & \text { if } x_{t}^{(1)}>\hat{\bar{c}} \\ 2 \hat{\hat{c}}-\left(x_{t}^{(1)}\right) & \text { if } x_{t}^{(1)}<\hat{\hat{c}} \\ x_{t}^{(1)} & \text { otherwise }\end{cases}
$$

If necessary, continue reflecting each subsequent series in the same way as $x^{(2)}$ until the series is completely within the desired bounds $[\underline{c}, \bar{c}]$. Call this series $x^{(R)}$ for some $R \geq 1$ (Figure 3 ) and (Figure 4).

Lemma 4: For some $R \geq 1, x_{[T t]}^{(R)} \Rightarrow B_{d+1}^{c, \bar{c}}(t)$ as $T \rightarrow \infty$, where $B_{d+1}^{c, \bar{c}}(t)$ is a now a reflected fractional Brownian motion.

Proof : For some $R \geq 1, x^{(R)} \in[\underline{c}, \bar{c}]$. The result then follows from the reflection principle for fractional Brownian motions. See Lee (2011).

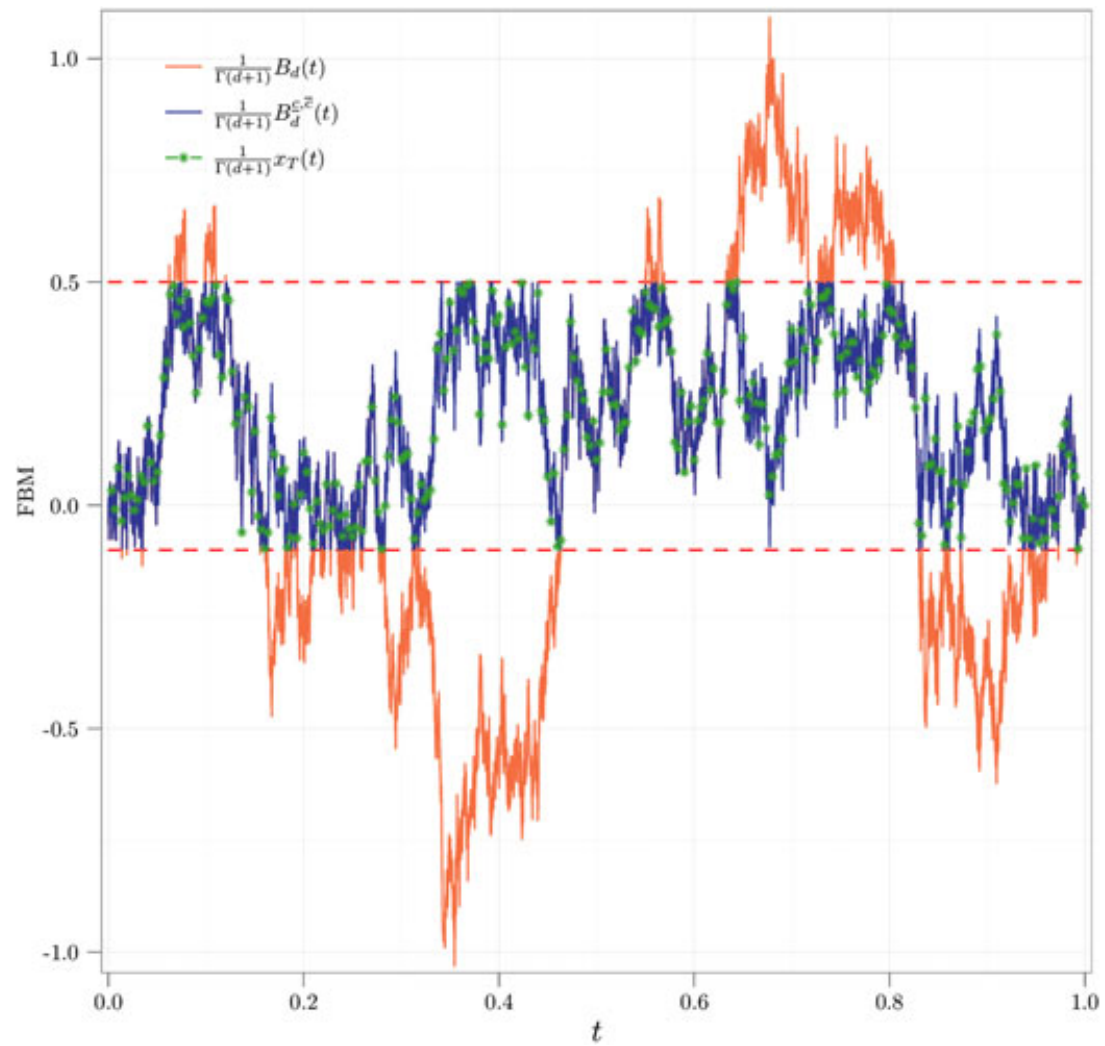

Figure 4. Regulated fractional Brownian motions (FBM): the graph is generated with a sample of size $2^{13}, d=-0.1$, and asymptotic reflective distributional bounds $\underline{c}=-0.1$ and $\bar{c}=0.5$ 
What Lemma 4 implies is that the choice of the algorithm does not alter the asymptotic results of this article. In fact, it goes so far as to say that both algorithms produce series that tend to the same limiting distribution but with different interpretation of the barriers. In the first algorithm, the two reflecting barriers are also full absorption barriers. This means that any value of the series outside the reflective barrier is indeed reflected but simultaneously absorbed by it as well (Figure 1) and (Figure 2). In the second algorithm, the reflecting barriers act as one would intuitively expect them to, namely fully reflecting the series path to produce shadow values that are probabilistically equivalent to the original paths (Figure 3) and (Figure 4).

More importantly, the aforementioned simulation experiments demonstrate that the cadlag space approximation of the series obtained by iteration using one of the algorithms presented earlier tends in distribution to the appropriate type II regulated fractionally integrated Brownian motion path. This is precisely Theorem 1 of this article.

\section{CONCLUSION}

The aim of this article was to extend the idea of a bounded integrated series to bounded integrated series with fractional integration orders. To this end, this article has established that the limiting distribution of such series is a regulated (bounded) type II fractional Brownian motion. Note that the type II nature of this limiting distribution arises because of considerations that in practice, only series with positive time indices are tractable. Moreover, the results in this article are a natural extension of the work presented in Cavaliere and Xu (2011). In principle, this work meshes the idea behind the latter article with the results on the asymptotics for fractionally integrated processes presented in Wang et al. (2002). The results from the latter article were chosen particularly for their weak assumptions. In fact, the conditions under which the results presented here hold are weaker than those required by Cavaliere and Xu (2011).

This article, like its predecessors, has not addressed the issue of unknown bounds or of regulated trending series. Although a discussion of the latter problem can be read in Carrion-i Silvestre and Gadea (2010), Cavaliere and Xu (2011) do present a rather simple solution that should not be difficult to implement. In fact, their solution is likely to hold in the setting of this article with slight modifications. This is research that is being undertaken. As far as unknown bounds are concerned, it is difficult to imagine why the results presented here would fail to hold in the presence of consistent estimators. What remains to be shown however is how one might go about obtaining these. Furthermore, this article has also not concerned itself with estimating the fractional integration parameter $d$. There is a growing literature on how to obtain consistent estimates of this parameter, and it should be a relatively straightforward task to extend the content of the current work to reflect that.

In closing, one ought to consider that the aim of this article was to extend an idea by meshing it with another. For this reason, this work has not addressed empirical implications one might find in Cavaliere and $\mathrm{Xu}$ (2011), although simulation evidence was presented in the preceding section. This however is intentional, and an article applying the ideas of this article to unit root testing is in the process of being written. However, perhaps the more interesting contribution here is that it is a step closer to developing a theory for regulated co-integrated series. One hopes that the results presented herein will stimulate future research in this area.

\section{REFERENCES}

Billingsley, P. (1968) Convergence of Probability Measures. New York: Wiley.

Carrion-i Silvestre, J. L. and Gadea, M. D. (2010) Bounds, breaks and unit root tests.

Cavaliere, G. (2005) Limited time series with a unit root. Econometric Theory 21(05), 907-45.

Cavaliere, G. and Xu, F. (2011) Testing for unit roots in bounded time series. Journal of Econometrics. http://www2.stat.unibo.it/cavaliere/research.html Chang, Y. and Park, J. Y. (2002) On the asymptotics of adf tests for unit roots. Econometric Reviews 21(4), 431-47.

Davidson, J. and Hashimzade, N. (2009) Type I and type II fractional Brownian motions: a reconsideration. Computational Statistics \& Data Analysis 53(6), 2089-106. Elliott, G., Rothenberg, T. J. and Stock, J. H. (1996) Efficient tests for an autoregressive unit root. Econometrica 64(4), 813-36.

Granger, C. W. J. (2010) Some thoughts on the development of cointegration. Journal of Econometrics 158(1), 3-6.

Harrison, J. M. (1985) Brownian Motion and Stochastic Flow Systems. New York: Wiley.

Lee, C. (2011) On the return time for a reflected fractional Brownian motion process on the positive orthant. Journal of Applied Probability 48(1), 145-53. Marinucci, D. and Robinson, P. M. (2000) Weak convergence of multivariate fractional processes. Stochastic Processes and their applications 86(1), 103-20.

Wang, Q., Lin, Y. X. and Gulati, C. (2002) Asymptotics for general nonstationary fractionally integrated processes without prehistoric influence. Journal of Applied Mathematics and Decision Sciences 6(4), 255-69.

\section{APPENDIX A}

Proof of Theorem 1 : The proof follows the strategy outlined in Cavaliere and Xu (2011). In this regard, consider the following process:

$$
\bar{x}_{t}= \begin{cases}\bar{b} & \text { if } \widetilde{x}_{t-1}+z_{t}>\bar{b} \\ \underline{b} & \text { if } \widetilde{x}_{t-1}+z_{t}<\underline{b} \\ \widetilde{x}_{t-1}+z_{t} & \text { otherwise }\end{cases}
$$

and assume that $\widetilde{x}_{0}=x_{0}=0$. This implies that the process can be recursively defined as follows: 


$$
\begin{aligned}
\widetilde{x}_{t} & =\widetilde{x}_{0}+\sum_{i=1}^{t} z_{i}+\sum_{i=1}^{t} \Delta_{+}^{-d} \underline{\underline{\xi}}-\sum_{d, i}^{t} \Delta_{i=1}^{-d} \bar{\xi}_{d, i} \\
& =\widetilde{x}_{0}+\sum_{i=1}^{t} \Delta_{+}^{-d} v_{i}+L_{t}-Q_{t} \\
& =\widetilde{x}_{0}+V_{t}+L_{t}-Q_{t} .
\end{aligned}
$$

First, remark that the second line above holds since $z_{t}=\Delta_{+}^{-d} v_{t}$. Further, note that the aforementioned sequence is in fact the Harrison (1985) construction of a regulated stochastic process, but it is not continuous. Thus, if we can obtain a continuous approximation of the aforementioned sequence on the $\mathcal{C}[0,1]$ space with a uniform metric and show convergence, convergence in the original $\mathcal{D}[0,1]$ space will follow by Theorem 4.1 in Billingsley (1968). To this end, there is no loss of generality in setting $\underline{c}=0$.

Moreover, a closer look at the aforementioned construction implies that

$$
\begin{aligned}
& \Delta_{+}^{-d} \underline{\xi}_{d, i}=-\left(\widetilde{x}_{t-1}+\Delta_{+}^{-d} v_{t}\right) \\
& \Delta_{+}^{-d} \underline{\xi}_{d, i}=-\left(\widetilde{x}_{t-1}+\Delta_{+}^{-d} v_{t}-\bar{c} \kappa(d) T^{(d+1 / 2)}\right) .
\end{aligned}
$$

Now, because $\underline{\xi}_{d, t}=\Delta_{+}^{d} \underline{\xi}_{t}$ and $\bar{\xi}_{d, t}=\Delta_{+}^{d} \bar{\xi}_{t}$, this implies that the regulators for the RFI(d) process $x_{t}$ defined in eqns (3)-(6) are in fact $\underline{\xi}_{t}$ and $\bar{\xi}_{t}$, as desired.

Now apply the broken line process to $\widetilde{x}_{t}$ and create its continuous approximant on $\mathcal{C}[0,1]$ as follows:

$$
\widetilde{x}_{T}(t)=\left(\kappa^{2}(d) T^{2(d+1 / 2)}\right)^{-1 / 2} \widetilde{x}_{[T t]} .
$$

Do the same for the other terms:

$$
\begin{aligned}
& V_{T}(t)=\left(\kappa^{2}(d) T^{2(d+1 / 2)}\right)^{-1 / 2} \sum_{i=1}^{[T t]} \Delta_{+}^{-d} v_{i}+\Delta_{+}^{-d} v_{[T t]+1}\left(\frac{T t-[T t]}{\kappa(d) T^{(d+1 / 2)}}\right)
\end{aligned}
$$

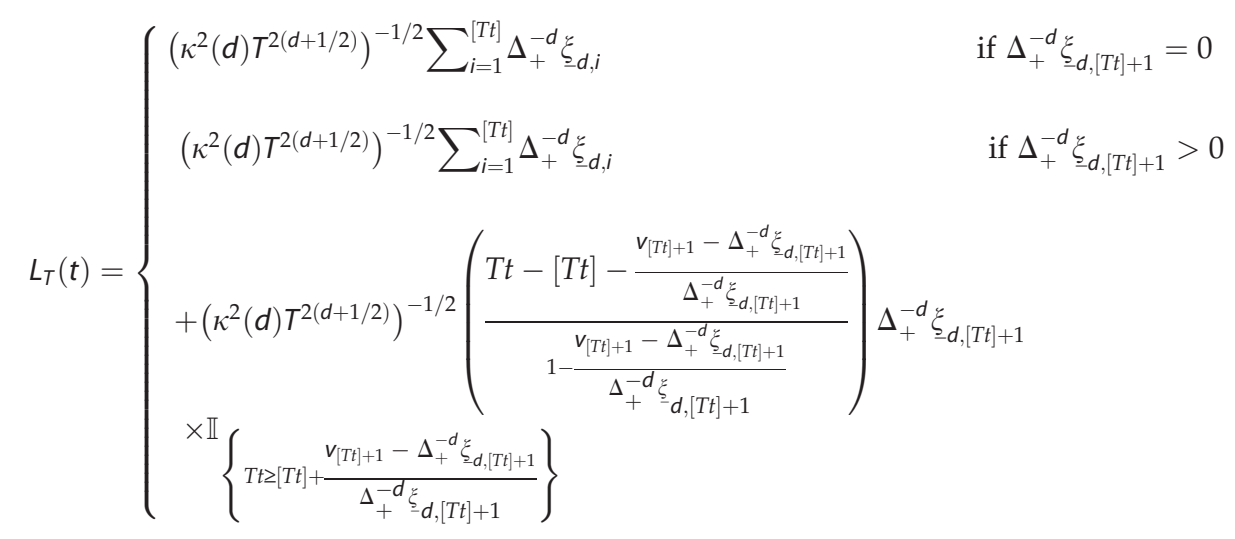

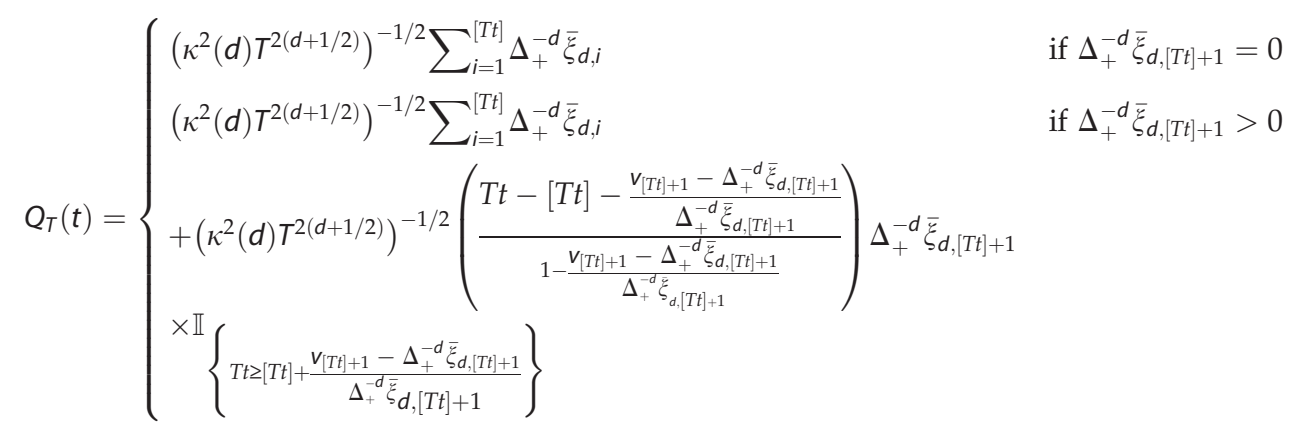

The principal idea here is that the aforementioned construction is a continuous version of the Harrison (1985) construction of a regulated process. More important, notice that by Lemma 2 and the continuous mapping theorem $(C M T), V_{T}(t) \Rightarrow B_{d+1}(t)$, in other words, a type II fractional Brownian motion with parameter $d$. In fact, by applying the CMT to $\widetilde{x}_{t}(t)$, the limiting result is $\widetilde{x}_{T}(t)=B_{d+1}^{0, \bar{c}}(t)+L_{T}(t)-Q_{t}(t)$. Because of Proposition 2.4.6 in Harrison (1985), this limiting result is the 'unique' such function that guarantees that $\widetilde{x}_{T}(t) \in[0, \bar{c}]$. In other words, by Definition 2 , the limiting distribution of $\widetilde{x}_{T}(t)$ is a regulated type II fractionally 
integrated Brownian motion, as desired. What is left to show is that the aforementioned construction converges in $\mathcal{D}[0,1]$. To this end, consider the following difference:

$$
\begin{aligned}
x_{T}(t)-\left(\kappa^{2}(d) T^{2(d+1 / 2)}\right)^{-1 / 2} x_{[T t]}= & \left(L_{T}-L_{[T t]}\right)-\left(Q_{T}-Q_{[T t]}\right) \\
& +\left(\kappa^{2}(d) T^{2(d+1 / 2)}\right)^{-1 / 2} \Delta_{+}^{-d} v_{[T t]+1}(T t-[T t]) .
\end{aligned}
$$

Because both $\left|L_{T}-L_{[T t]}\right|$ and $\left|Q_{T}-Q_{[T t]}\right|$ are smaller than $\left(\kappa^{2}(d) T^{2(d+1 / 2)}\right)^{-1 / 2}\left|\Delta_{+}^{-d} V_{[T t]+1}\right|$, it follows that

$$
\left|x_{T}(t)-\left(\kappa^{2}(d) T^{2(d+1 / 2)}\right)^{-1 / 2} x_{[T t]}\right| \leq 2\left(\kappa^{2}(d) T^{2(d+1 / 2)}\right)^{-1 / 2}\left|\Delta_{+}^{-d} v_{[T t]+1}\right| .
$$

Thus,

$$
\sup _{t \in[0,1]}\left|\widetilde{x}_{T}(t)-\left(\kappa^{2}(d) T^{2(d+1 / 2)}\right)^{-1 / 2} \widetilde{x}_{[T t]}\right| \leq 2\left(\kappa^{2}(d) T^{2(d+1 / 2)}\right)^{-1 / 2}\left|\max _{t=1, \ldots, T} \Delta_{+}^{-d} v_{t}\right| .
$$

The proof of Theorem 1 in Wang et al. (2002) shows that under the conditions of this article, $\left|\max _{t=1, \ldots, T} \Delta_{+}^{-d} v_{t}\right|$ is $o_{P}\left(T^{(d+1 / 2)}\right)$. In turn, this implies that

$$
\sup _{t \in[0,1]}\left|\widetilde{x}_{T}(t)-\left(\kappa^{2}(d) T^{2(d+1 / 2)}\right)^{-1 / 2} \widetilde{x}_{[T t]}\right| \rightarrow p 0
$$

If it is the case that $\sup _{t}\left|x_{T}(t)-\widetilde{x}_{T}(t)\right| \rightarrow_{p} 0$, the result follows. Indeed, Lemma 4 and assumption (d) imply the needed condition and $x_{T}(t) \Rightarrow B_{d+1}^{[0, \bar{c}]}(t)$.

Lemma 5: Let $\left\{x_{t}\right\}$ and $\left\{\widetilde{x}_{t}\right\}$ be defined as above. Then,

$$
\begin{aligned}
\max _{t=0, \ldots, T}=\left|x_{t}-\widetilde{x}_{t}\right| & \leq \max \left(\max _{t=0, \ldots, T} \Delta_{+}^{-d} \underline{\xi}_{d, t}, \max _{t=0, \ldots, T} \Delta_{+}^{-d} \bar{\xi}_{d, t}\right) \\
& \leq \max _{t=0, \ldots, T} \Delta_{+}^{-d} \underline{\xi}_{d, t}+\max _{t=0, \ldots, T} \Delta_{+}^{-d} \bar{\xi}_{d, t} .
\end{aligned}
$$

Proof : This is in fact just a version of Lemma 7 in Cavaliere (2005). The proof is virtually identical as that found there. 